\title{
PREDICTORS OF ORAL INTAKE DIFFICULTY IN OLDER PATIENTS WITH DYSPHAGIA
}

\author{
Y. Kuroda
}

\begin{abstract}
The aim of this study was to identify the predictors of oral intake difficulty in older patients with dysphagia. The subjects were 133 hospitalized older individuals with dysphagia. They were divided into two groups according to the oral intake ability: the oral intake and non-oral intake groups. Swallowing function was measured with a graded water swallowing test and a food intake level scale. Functional status was evaluated with a physical dependency scale and a comprehension scale. Nutritional status was measured with anthropometric measures and serum albumin levels. The non-oral intake group was significantly impaired in the swallowing measures, physical dependency, and mid-upper arm circumference. Multivariate analysis revealed that impairment in mid-upper arm circumference and graded water swallowing test were independently associated with oral intake difficulty. In conclusion, swallowing impairment, advanced body mass reduction, and physical dependency were the significant predictors of oral intake difficulty in older patients with dysphagia.
\end{abstract}

Key words: Dysphagia, elderly, malnutrition, oral intake, physical dependency.

\section{Introduction}

Dysphagia is a highly prevalent condition in hospitalized older adults. Recent studies have reported a prevalence of $27 \%$ in independently-living elderly (1) and $47 \%$ in hospitalized elderly (2). One major consequence of dysphagia is reduced oral intake due to swallowing difficulty. A recent large-scale study demonstrated that $41 \%$ of older patients admitted with aspiration pneumonia could not achieve sufficient oral intake within 30 days (3). This is a serious issue in current geriatric medicine, because older patients with poor oral intake often become candidates to receive long-term artificial nutrition, which may lead to diminished quality of life. Therefore, it is important to identify the predictors of oral intake difficulty in this clinical population. Given that previous studies demonstrated that dysphagia in the elderly was associated with older age, dementia, impaired activity of daily living, and malnutrition $(2,4$, $5)$, those factors may affect the oral intake. The aim of this study was to identify the predictive factors for poor oral

Department of Rehabilitation, St. Francis Hospital, 9-20 Komine-machi, Nagasaki City, 852-8125 Japan

Corresponding Author: Yoshitoshi Kuroda, Department of Rehabilitation, St. Francis Hospital, Nagasaki, Japan, 9-20 Komine-machi, Nagasaki City, 852-8125 Japan, Telephone: +81-95-846-1888, Fax: +81-95-845-7600, Email: kuroda@athena.ocn. ne.jp intake in older patients with dysphagia.

\section{Methods}

The subjects of this study were older individuals admitted to St. Francis Hospital for acute care between April 2012 and February 2014. The study inclusion criteria were aged 65 and older and referral to the Speech Pathology Service for assessment of dysphagia. Six patients with malignancy, four patients already being tube-fed, and one patient who had cerebral hemorrhage during the hospitalization were excluded to avoid their influence on the results. A total of 133 patients were enrolled for this study.

Swallowing measures consisted of a graded water swallowing test (GWST) (6) and a food intake level scale (FILS) (7). The GWST uses 2, 3, and $5 \mathrm{ml}$ of plain and thickened water in a graded manner, ranging from 0 (fails in $2 \mathrm{ml}$ thickened water trial) to 6 (passes in $5 \mathrm{ml}$ plain water trial). The FILS is an observer-rating scale for assessing the severity of dysphagia, examining to what degree patients take food orally on a daily basis, ranging from 0 (no oral intake, and no swallowing training) to 10 (normal oral food intake). Functional measures included a physical dependency scale and comprehension scale that are described elsewhere (6). Nutritional measures included mid-upper arm circumference (MUAC), calf 
circumference (CC), and serum albumin levels. MUAC was measured at the midpoint between the tip of the acromion process and the tip of the olecranon process of the left arm. CC was measured at the point of maximal circumference of the left leg. In principle, the evaluations were performed in the early days of hospitalization.

The subjects were divided into two groups according to the oral intake ability. The oral intake group $(n=91)$ were those who achieved sufficient oral intake (FILS of 7 or above) until the end of hospitalization. The nonoral intake group $(n=42)$ were those who continued to require artificial nutrition (FILS of 6 or below) during the hospitalization. In this study, patients of the nonoral intake group were defined as those with oral intake difficulty. To make the data more readily comparable and to accommodate the logistic regression analysis, ordinal and cardinal variables were converted into the categorical measures using cutoff points. The cutoff points were applied for age (85 years or older), GWST (score $<4$ ), FILS (score $<5$ ), physical dependency scale (score $<1$ ), comprehension scale (score $<2)$, MUAC $(<22.5 \mathrm{~cm}$ for men and $<20.8 \mathrm{~cm}$ for women), CC $(<31.3 \mathrm{~cm}$ for men and $<29.9 \mathrm{~cm}$ for women), and albumin levels $(<2.8 \mathrm{~g} /$ $\mathrm{dL}$ ). The cutoff points of MUAC and CC were 2 standard deviations below the means for a normal population aged 40-44 years for each sex (8), and the other cutoff points were arbitrary chosen close to the mean.

Comparative analyses between the two groups were performed using the Student's t-test, Mann-Whitney test, and chi square test. Multivariate logistic regression analysis was performed using the oral intake difficulty represented by the non-oral intake group as the dependent variable and other dichotomous variables as independent variables. A p-value $<0.05$ was considered statistically significant. This study was approved by the ethics committee of our hospital.

\section{Results}

The basic results are shown in Table 1 . The subjects were 53 men and 80 women, with a mean \pm SD age of $85.8 \pm 6.2$ years. Primary diseases on admission were respiratory disease $(n=88)$, digestive disease $(n=$ 12), urinary infection $(n=6)$, cardiac disease $(n=5)$, orthopedic disease $(n=4)$, cerebrovascular accident (n $=3)$, and others $(n=15)$. Of the 133 patients, 88 had dementia, and 58 had a history of other neurological disease (cerebrovascular disease or Parkinson's disease) according to the medical records.

A comparison of the variables between the oral intake and non-oral intake groups is shown in Table 2. There were no significant differences between the two groups in age, sex, or disease conditions. Regarding the swallowing measures, the non-oral intake group showed significantly higher rates of impaired GWST $(\mathrm{p}<0.001)$ and impaired FILS $(\mathrm{p}<0.001)$ compared with the oral intake group. The non-oral intake group also showed significantly higher rates of physical dependency $(p=0.005)$, but not impaired comprehension $(\mathrm{p}=0.593)$. Regarding nutritional status, the non-oral intake group showed significantly higher rates of decreased MUAC $(\mathrm{p}<0.001)$, but not decreased CC $(p=0.231)$ or low serum albumin levels $(p=0.139)$.

Table 1

Basic results

\begin{tabular}{lc}
\hline Evaluation items & $\begin{array}{c}\text { Mean } \pm \text { SD (range) } \\
\text { or number (\%) }\end{array}$ \\
\hline Age (years) & $85.8 \pm 6.2(66-100)$ \\
Gender (male) & $53(40 \%)$ \\
Respiratory disease & $88(66 \%)$ \\
Dementia & $88(66 \%)$ \\
History of neurological disease & $58(44 \%)$ \\
Graded water swallowing test (0-6) & $3.9 \pm 2.1(0-6)$ \\
Food intake level scale (1-10) & $5.1 \pm 1.8(2-9)$ \\
Physical dependency scale (0-7) & $1.0 \pm 1.4(0-7)$ \\
Comprehension scale (0-5) & $2.4 \pm 1.2(0-5)$ \\
Mid-upper arm circumference (cm) & $19.9 \pm 3.1(13.6-28.2)$ \\
Calf circumference (cm) & $24.4 \pm 3.6(15.8-33.2)$ \\
Serum albumin level (g/dL) & $2.8 \pm 0.5(1.3-4.3)$ \\
\hline
\end{tabular}

The result of multivariate regression analysis showed that the significant and independent predictors of oral intake difficulty were decreased MUAC (odds ratio $=4.67 ; 95 \%$ confidence interval: $1.49,14.70 ; \mathrm{p}=0.008)$ and impaired GWST (odds ratio $=3.62 ; 95 \%$ confidence interval: $1.63,8.07 ; \mathrm{p}=0.002)$.

\section{Discussion}

Of the 113 patients, 91 achieved sufficient oral intake until the end of hospitalization whereas 42 did not. No significant differences were observed in demographic variables (age and sex) or disease conditions between the oral intake and non-oral intake groups. Considering that the prevalence of dementia and other neurological conditions was not significantly different between the two groups, it was unlikely that the oral intake difficulty in the present subjects was related to such neurological conditions.

The results showed that impaired GWST, impaired FILS, physical dependency, and decreased MUAC were the significant predictors of poor oral intake. The independent association between decreased MUAC and poor oral intake suggests a non-negligible influence of significant body mass loss on oral intake behavior in older patients with dysphagia. Considering that some authors have suggested that MUAC, more so than CC, reflects end-stage decline (9) and that CC is seemingly 
Table 2

Comparison between the oral intake and non-oral intake groups

\begin{tabular}{|c|c|c|c|c|}
\hline Variables & Total $(n=133)$ & Oral intake $(n=91)$ & Non-oral intake $(n=42)$ & p-value \\
\hline Sex (male), n (\%) & $53(40 \%)$ & $33(36 \%)$ & $20(48 \%)$ & 0.263 \\
\hline Respiratory disease, n (\%) & $88(66 \%)$ & $57(63 \%)$ & $31(74 \%)$ & 0.206 \\
\hline Dementia, n (\%) & $88(66 \%)$ & $59(65 \%)$ & $29(69 \%)$ & 0.633 \\
\hline History of neurological disease, $\mathrm{n}(\%)$ & $58(44 \%)$ & $43(47 \%)$ & $15(36 \%)$ & 0.212 \\
\hline Impaired FILS**, n $(\%)$ & $38(29 \%)$ & $17(19 \%)$ & $21(50 \%)$ & $<0.001$ \\
\hline Physical dependency $(<1)$, n (\%) & $78(59 \%)$ & $46(51 \%)$ & $32(76 \%)$ & 0.005 \\
\hline Impaired comprehension $(<2), \mathrm{n}(\%)$ & $31(23 \%)$ & $20(22 \%)$ & $11(26 \%)$ & 0.593 \\
\hline Decreased MUAC ${ }^{* * *}, \mathrm{n}(\%)$ & $96(72 \%)$ & $58(64 \%)$ & $38(91 \%)$ & 0.001 \\
\hline
\end{tabular}

*GWST: graded water swallowing test (impaired: $<4$, failure with the $3 \mathrm{~mL}$ plain water trial); **FILS: food intake level scale (impaired: $<5$, difficulty in consuming a

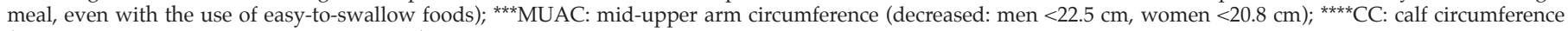
(decreased: men $<31.3 \mathrm{~cm}$, women $<29.9 \mathrm{~cm}$ )

affected by ambulatory status, a significant reduction in MUAC may be a better indicator of profound changes in body mass compared with that in CC. Therefore we postulated here that advanced body mass reduction was associated with oral intake difficulty. One hypothesis is that general and profound reduction of muscle mass and function extends into the head and neck region, which leads to the disruption of the swallowing mechanism, resulting in impaired efficiency and safety of swallowing. This hypothesis can be explained based on the concept of sarcopenic dysphagia (10). Another hypothesis is that profound malnutrition represented by decreased MUAC may be associated with adverse conditions including delay in recovery from illness, susceptibility to infections, and increased comorbidity, fatigue, and psychological distress such as apathy and depression (11, 12), all of which may affect oral intake. We consider that these two hypotheses are not mutually exclusive, and further data are needed to support each hypothesis.

The major limitations of the present study are as follows. First, instrumental assessment of muscle mass and function was not conducted, which may limit the discussion. Second, this was a single-institution study, which may limit generalization from the results.

In conclusion, swallowing impairment, advanced body mass reduction, and physical dependency were the significant predictors of poor oral intake in older patients with dysphagia. Further investigations on the underlying mechanism of the condition are required.

Conflicts of interest: The author has no conflict of interest to disclosure, and do not receive any funding for this study

\section{References}

1. Serra-Prat M, Hinojosa G, López D et al. Prevalence of oropharyngeal dysphagia and impaired safety and efficacy of swallow in independently living older persons. J Am Geriatr Soc 2011; 59: 186-187.

2. Carrión S, Cabré M, Monteis R et al. Oropharyngeal dysphagia is a prevalent risk factor for malnutrition in a cohort of older patients admitted with an acute disease to a general hospital. Clin Nutr 2015; 34: 436-442.

3. Momosaki R, Yasunaga H, Matsui H, Horiguchi H, Fushimi K, Abo M. Predictive factors for oral intake after aspiration pneumonia in older adults. Geriatr Gerontol Int (Epub ahead of print).

4. Cabré M, Serra Prat M. Force L, Almirall J, Palomera E, Clavé P. Oropharyngeal dysphagia is a risk factor for readmission for pneumonia in the very elderly persons: observational prospective study. J Gerontol A Biol Sci Med Sci 2014; 69: 330-337.

5. Kuroda Y. Clinical characteristics associated with dysphagia in the hospitalized elderly. J Aging Res Clin Pract 2015; 4: 133-136.

6. Kuroda Y. Relationship between swallowing function, functional and nutritional status in hospitalized elderly individuals. Int J Speech Lang Pathol Aud 2014; 2: 20-26.

7. Kunieda K, Ohno T, Fujishima I, Hojo K, Morita T. Reliability and validity of a tool to measure the severity of dysphagia: the food intake level scale. J Pain Symptom Manage 2013; 46: 201-206.

8. Hosoya N, Okada T, Muto Y et al. Japanese anthropometric reference data 2001 (JARD2001). Jpn J Nutr Assess 2002;19(Suppl): 1-81. (Japanese)

9. Dent E, Chapman I, Piantadosi C, Visvanathan R. Nutritional screening tool and anthropometric measures associate with hospital discharge outcomes in older people. Austral J Ageing 2015; 34:E1-6.

10. Wakabayashi H. Presbyphagia and sarcopenic dysphagia: association between aging, sarcopenia, and deglutition disorders. J Frail Aging 2014; 3: 97-103.

11. Norman K. Richard C, Losch H, Pirlich M. Prognostic impact of diseaserelated malnutrition. Clin Nutr 2008; 27: 5-15.

12. Saunders J, Smith T. Malnutrition: causes and consequences. Clinical Medicine 2010; 10: 624-627. 